American Journal of Neuroscience 2 (1): 48-58, 2011

ISSN 1948-9900

(C) 2011 Science Publications

\title{
Functional Neuroimaging in Dementia and other Amnesic Disorders: A Radiological Review
}

\author{
${ }^{1}$ Leonardo Caixeta, ${ }^{1}$ Renata Teles Vieira, ${ }^{3,4}$ Sergio Machado, \\ ${ }^{2}$ Bruno Galafassi, ${ }^{1}$ Ciro Vargas and ${ }^{1}$ Lorena Resende \\ ${ }^{1}$ Neuropsychiatric Cognitive Unit of the Hospital das Clinicas of the Federal, \\ University of Goias, Brazil \\ ${ }^{2}$ Neuroimaging Unit, Imaging Diagnostic Center, Goiania-GO, Brazil \\ ${ }^{3}$ Institute of Psychiatry, Panic and Respiration, \\ Federal University of Rio de Janeiro (IPUB/UFRJ), Brazil \\ ${ }^{4}$ Institute of Psychiatry, Brain Mapping and Sensory Motor Integration, \\ Federal University of Rio de Janeiro (IPUB/UFRJ), Brazil
}

\begin{abstract}
Problem statement: The structural and functional neuroimaging have much to contribute to the cognitive neuroscience. Approach: We describe a radiological review of the major dementia syndromes and Mild Cognitive Impairment (MCI), included Alzheimer's disease, Lewy body dementia, Vascular dementia, Front temporal Lobar degeneration, Dementia associated with parkinsonism and other amnesic syndromes. Results: The different syndromes and diseases presenting with dementia have different patterns of brain perfusion abnormalities, it is possible to distinguish them with good specificity with neuroimaging. Conclusion: New imaging techniques carry the hope of revolutionizing the diagnosis of dementia so as to obtain a complete molecular, structural and metabolic characterization, which could be used to improve diagnosis and to stage each patient and follow disease progression and response to treatment. Structural and functional imaging modalities contribute to the diagnosis and understanding of the different dementias.
\end{abstract}

Key words: Dementia, mild cognitive impairment, functional neuroimaging, Computed Tomography (CT), Magnetic Resonance Imaging (MRI), functional Magnetic Resonance Imaging (fMRI), Electroencephalography (EEG), Working Memory (WM)

\section{INTRODUCTION}

Neuroimaging has revolutionized the field of cognitive neuroscience. Early studies of brain-behavior relationships relied on a precise neurological examination as the basis for hypothesizing the site of brain damage that was responsible for a given behavioral syndrome. Episodic amnesia, for example, clearly implicated the hippocampus as the site of recent memory abilities.

Clinicopathological correlations were the earliest means of obtaining precise data on the site of damage causing a specific neurobehavioral syndrome (D'Esposito, 2000; Pievani et al., 2011). In 1861, Paul Broca's observations of nonfluent aphasia in the setting of left inferior frontal gyrus damage cemented the belief that this brain region was critical for speech output (Burns and Fahy, 2010). The advent of structural brain imaging more than 100 years after Broca's observations, first with Computed Tomography (CT) and later with Magnetic Resonance Imaging (MRI), paved the way for more precise anatomical localization of the cognitive deficits that are manifest after brain injury (Fayed et al., 2006).

Anatomical analyses of Broca's aphasia using structural Neuroimaging have more precisely determined that damage restricted to the inferior frontal gyrus causes only a transient aphasia, with recovery within weeks to months (Dronkers, 1996; Cappa, 2011). Instead, damage to deep white matter and insular cortex causes persistent nonfluency. Noninvasive, structural neuroimaging provides the remarkable power to detail anatomical pathology in every stroke patient without relying upon the infrequently obtained autopsy.

Neuroimaging is a powerful tool for creative exploration of the epidemiology, diagnostic sensitivity, progression and therapeutic efficacy in many brain diseases featured by memory impairment (Herholz et

Corresponding Author: Leonardo Caixeta, Neuropsychiatric Cognitive Unit of the Hospital das Clinicas of the Federal, Federal University of Goias, Brazil Tel: 55-62-32047223 Fax: 55-62-32047223 
al., 2004). Some consider modern functional neuroimaging methods as useful tools to establish similarities and differences between different forms of amnesias with respect to their brain correlates, while others consider them adequate for constituting groups of patients in a research perspective, but still out of reach for the practitioner (Celsis, 2000; Pievani et al., 2011). Thus, we will describe a radiological review of the major dementia syndromes and Mild Cognitive Impairment (MCI), included Alzheimer's disease, Lewy body dementia, Vascular dementia, Frontotemporal Lobar degeneration, Dementia associated with parkinsonism and other amnesic syndromes.

Neuroimaging techniques: Functional neuroimaging broadly defined as techniques that provide measures of brain activity, has further increased our ability to study the neural basis of cognition and behavior. Functional neuroimaging dates back to the use of electrophysiological methods such as Electroencephalography (EEG) (Walters-Williams and $\mathrm{Li}, 2011)$. However, the lack of spatial resolution in these methods (i.e., often limited to hemispheric or anterior-posterior differences) did not allow testing hypotheses regarding the precise anatomical location of a brain region subserving a given cognitive process (D'Esposito, 2000; Pievani et al., 2011). The modern era of functional brain imaging, bringing markedly improved spatial resolution, was introduced first in the mid-1970s using the xenon cerebral blood flow technique and later in the mid-1980s using positron emission tomography (Mazziotta and Phelps, 1984; Petersen et al., 1988; Rossini and Forno, 2004). In more recent years, functional Magnetic Resonance Imaging (fMRI) has rapidly emerged as an extremely powerful technique with many advantages over Positron Emission Tomography (PET) for studying cognition. In dementia evaluation, the most commonly used tracers are $99 \mathrm{mTc}$-HMPAO for SPECT brain perfusion and 18F-FDG for PET glucose's metabolism. For practical purposes, the clinical analysis of brain perfusion and glucose metabolism are equivalent, so that hypo perfusion areas in brain SPECT roughly correspond to glucolytic hypo metabolism in PET. The different syndromes and diseases presenting with dementia have different patterns of brain perfusion abnormalities, so we can distinguish them with good specificity.

Normal aging: Brain aging is characterized by numerous physiological, structural, functional and neurocognitive changes. The interplay of these various processes is complex and characterized by large interindividual differences. The level of cognitive functioning achieved by a group of elderly is largely determined by the health of individuals within this group (Aine et al., 2011). Individuals with a history of hypertension, for example, are likely to have multiple white matter insults which compromise cognitive functioning, independent of aging processes. The health of the elderly group has not been well-documented in most previous studies and elderly participants are rarely excluded, or placed into a separate group, due to healthrelated problems (Caserta et al., 2009). In addition, recent results show that white matter tracts within the frontal and temporal lobes, regions critical for higher cognitive functions, continue to mature well into the 4 th decade of life (Aine et al., 2011). This suggests that a young age group may not be the best control group for understanding aging effects on the brain since development is ongoing within this age range.

Functional neuroimaging studies of cognitively intact older adults have consistently shown volume loss and loss of white matter structural integrity, particularly in prefrontal cortex, which may be associated with cognitive decline. For instance, age-related deficits in Working Memory (WM) and episodic memory abilities are related to changes in Prefrontal Cortex (PFC) function. Reviews of these neuroimaging studies have generally concluded that with age there is a reduction in the hemispheric specialization of cognitive function in the frontal lobes that may either be due to dedifferentiation of function, deficits in function and/or functional reorganization and compensation. While data are incomplete, another consistent finding has been a decline in other cognitive domains, such as arithmetic/numerical ability and perceptual speed. Alternatively, other cognitive functions such as verbal ability, word knowledge and semantic memory remain quite preserved even to old age (Caserta et al., 2009).

Some functional neuroimaging studies on normal aging showed the involvement of different patterns of activation in comparison with control populations, such as increased frontal activity. These differences have been interpreted as reflecting the implementation of compensatory processes (Kalpouzos et al., 2008). Some authors (Rajah and D'Esposito, 2005) argue that in normal ageing distinct PFC regions exhibit different patterns of functional change, suggesting that agerelated changes in PFC function are not homogeneous in nature. Specifically, these authors hypothesize that normal ageing is related to the differentiation of cortical function in a bilateral ventral PFC and deficits in function in right dorsal and anterior PFC. As a result of these changes, functional compensation in left dorsal and anterior PFC may occur. 
Factors identified for healthy cognitive aging are multifactorial and likely incorporate biological systems as well as cognitive reserve (i.e., the capability of an individual to cope with a task in order to optimize the performance by the recruitment of different neural networks and/or by using alternative cognitive strategies).

Mild Cognitive Impairment (MCI): Mild Cognitive Impairment (MCI), a transitional state between normal aging and dementia, carries a four- to sixfold increased risk of future diagnosis of dementia. As complete druginduced reversal of $\mathrm{AD}$ symptoms seems unlikely, researchers are now focusing on the earliest stages of $\mathrm{AD}$ where a therapeutic intervention is likely to realize the greatest impact. Recently neuroimaging has received significant scientific consideration as a promising in vivo disease-tracking modality that can also provide potential surrogate biomarkers for therapeutic trials (Apostolova and Thompson, 2008).

Evidence to date indicates that functional brain decline precedes structural decline in prodromal dementia, including adults with MCI (Jak et al., 2009). Therefore, functional neuroimaging techniques may offer the unique ability to detect early functional brain changes in at-risk adults and identify the neurophysiologic markers that best predict dementia conversion.

While several volumetric techniques laid the foundation of the neuroimaging research in $\mathrm{AD}$ and MCI, more precise computational anatomy techniques have recently become available. This new technology detects and visualizes discrete changes in cortical and hippocampal integrity and tracks the spread of AD pathology throughout the living brain. Related methods can visualize regionally specific correlations between brain atrophy and important proxy measures of disease such as neuropsychological tests, age of onset or factors that may influence disease progression (Apostolova and Thompson, 2008).

Given that AD neuropathology preferentially targets the Medial Temporal Lobe (MTL) early in the course of the disease, thereby resulting in the hallmark episodic memory decline and amnestic MCI is thought to represent prodromal $\mathrm{AD}$, the majority of fMRI studies of MCI involve memory processing (particularly encoding) in amnesic samples (Jak et al., 2009). No known fMRI studies have been published focusing on other clinical subtypes of MCI. While several studies demonstrate increased Blood Oxygen Level Dependent (BOLD) response in the MTL (Dickerson et al., 2004; Hamalainen et al., 2007; Kircher et al., 2007; Sperling, 2007; Jak et al., 2009) others report decreased MTL activity in MCI (Johnson et al., 2006; Mandzia et al., 2009). These discrepant findings have been interpreted as reflecting bimodal functional activity whereby less impaired MCI subjects show increased BOLD response in the hippocampus corresponding to a slight or moderate neuronal dysfunction and more impaired MCI subjects demonstrate decreased BOLD response similar to the levels observed in mild AD patients as the cortical neuronal networks become more severely impaired with greater disease progression (Dickerson et al., 2004; Johnson et al., 2006; Hamalainen et al., 2007). However, this interpretation is primarily derived from cross-sectional studies and can only adequately be tested with longitudinal designs.

Few longitudinal fMRI studies of MCI have been reported. Although these studies are often limited by small sample sizes, they demonstrate promise for the use of fMRI to detect early AD. Those MCI patients who converted to AD showed a stronger relationship between brain activity in the left superior parietal lobe and the left precuneus during an angle discrimination task in the context of comparable performance (Vannini et al., 2007). Similarly, despite equivalent memory performance, Dickerson et al. (2004) reported that MCI patients who subsequently declined during a 2.5-year follow-up period demonstrated increased right parahippocampal gyros activity during picture encoding. In a more recent study, the same research group reported increased hippocampal activation predicted greater degree and rate of cognitive decline during a 6-year follow-up period, even after controlling for baseline level of impairment (Miller et al., 2008).

Mandzia et al. (2009) reported that MTL activation during recognition was positively correlated with behavioral performance. However, unlike their healthy peers, MCI adults did not show a strong relationship between MTL activity during picture encoding and subsequent retrieval success, highlighting the complexity of the relationship between BOLD signal and effectiveness of encoding strategies. In contrast, Johnson et al. (2006) found reduced BOLD signal change in the right hippocampus during picture encoding and in the posterior cingulated during recognition of learned items in an amnesic MCI group despite comparable performance to their healthy peers. However, when activation corresponding only to successfully learned words was examined, an increase in hippocampal activity was seen, suggesting that an increase in MTL activity may support successful memory encoding (Kircher et al., 2007). Similarly, a positive correlation between extent of parahippocampal and hippocampal activation and memory performance was found in MCI but, in a paradoxical fashion, greater clinical impairment, was also associated with 
recruitment of a larger region of the right parahippocampal gyrus during encoding (Dickerson et al., 2004). Data from Johnson et al. (2004) provided further evidence for hippocampal dysfunction in MCI, suggesting that adults with MCI do not habituate to increasingly familiar items in the same manner as healthy older adults who show expected reductions in BOLD response to repeated items over time.

Despite the prevalence of studies examining medial temporal cortex function supporting memory, other cortical areas have also been implicated in MCI. For example, a reduction in functional activity in the Posterior Cingulated Cortex (PCC) during recognition and episodic retrieval of previously learned line drawings (Johnson et al., 2006) and object working memory, but not during self-appraisal, has implicated this region in the memory retrieval difficulty seen in amnesic MCI. The degradation of PCC functioning in MCI is not surprising given that PET metabolic alterations in the temporoparietal cortices and in the posterior cingulated have been reported in MCI and AD as well as in no demented young and middle-aged adults at genetic risk for AD. Similarly, dedifferentiation in the retrosplenial cortex during the retrieval of recent versus remote autobiographical memories and during episodic versus semantic memory retrieval has been reported in amnesic MCI, further implicating the medial posterior cortex in MCI. Additionally, the neural substrates of visual working memory, self-appraisal and emotional working memory in MCI have also been examined and generally implicate a greater number of cortical regions (Jak et al., 2009). However, results are varied and highlight the need for greater attention to other cognitive processes in MCI in order to more fully understand changes in cortical functioning that may signal impending cognitive decline.

Dementias: Functional neuroimaging has many applications in the field of degenerative disorders (Van Heertum and Tikofsky, 2003; Herholz, 2003) and can even image amyloid plaques in the pre-mortem diagnosis of AD (Nordberg, 2004). The clinical identification and differential diagnosis of dementias is especially challenging in the early stages, but the need for early, accurate diagnosis has become more important, now that several medications for the treatment of mild to moderate Alzheimer's Disease (AD) are available. Many neurodegenerative diseases produce significant brain-function alterations detectable with PET or SPECT even when structural images with CT or MRI reveal no specific abnormalities (Silverman, 2004). Assessment of the precise diagnostic accuracy of PET had until recently been hindered by the paucity of data on diagnoses made using PET and confirmed by definitive histopathology examination. In the past few years, however, studies comparing neuropathology examination with PET have established reliable and consistent accuracy for diagnostic evaluations using PET-accuracies substantially exceeding those of comparable studies of the diagnostic value of SPECT or of both modalities assessed side by side, or of clinical evaluations done without nuclear imaging. Similar data are emerging concerning the prognostic value of (18) FFDG PET (Silverman, 2004). Improvements in the ability of PET to identify very early changes associated with $\mathrm{AD}$ and other neurodegenerative dementias are currently outpacing improvements in therapeutic options, but with advances in potential preventive and disease-modifying treatments appearing imminent, early detection and diagnosis will play an increasing role in the management of cementing illness.

Alzheimer's disease: $\mathrm{AD}$ is the commonest form of dementia worldwide. It manifests with relentlessly progressive cognitive decline presenting initially as memory loss and then spreads to affect all other cognitive faculties and the patients' ability to conduct an independent lifestyle (Degenszajn et al., 2001; Cheng and Zou, 2007; Lopes et al., 2011). Post-mortem examination reveals abundant cortical and hippocampal Neurotic Plaques (NP) and Neurofibrillary Tangles (NFT) as well as pancerebellar atrophy upon gross inspection of the brain. The neuronal degeneration pattern in Alzheimer's Disease (AD) spreads from the temporal medial areas to the posterior regions of the cingulated cortex and then to the parietal lobes and the rest of the temporal lobes, asymmetrically or not. Premortem, AD-associated brain changes can be clinically evaluated with the help of neuroimaging. They consist of global dysfunction with an early predilection for the hippocampal region and the temporo-parietal cortical areas (Devous, 2002; Mosconi et al., 2007). In advanced cases, the hypoperfusion pattern may reach the frontal lobes, but even in these cases the perfusion remains normal in thalamus, basal ganglia, motor cortex and occipital lobes (Silverman, 2004).

Among patients presenting with bilateral parietotemporal hypo perfusion, $82 \%$ have $\mathrm{AD}$, while the rest are due to Parkinson dementia or cerebrovascular disease. On the other hand, in patients with diagnosed $\mathrm{AD}, 100 \%$ have hypo concentration of 18F-FDG and $90 \%$ have parietal and temporal hypo perfusion with 99mTc-HMPAO (Lee et al., 2003). These typical findings are also found among those with early AD (Mosconi, 2005), while hippocampal hypo perfusion as only finding has low specificity for $\mathrm{AD}$ diagnosis. In 
individuals with mild cognitive impairment, the posterior cingulated hypo metabolism can predict who is going to develop $\mathrm{AD}$. However, not all patients with $\mathrm{AD}$ have Bilateral parietotemporal hypo perfusion. In a study conducted by our group in 104 patients fulfilling NINCDS-ADRDA criteria for probable AD, bilateral parietotemporal hypo perfusion was more frequent in patients with severe $\mathrm{AD}$, in those with early onset of the symptoms and in men (Nitrini et al., 2000).

Lewy body dementia: The metabolic defects described in this disease are very close to those found in $\mathrm{AD}$, but there is also hypo perfusion in the occipital lobes. One must have in mind that a patient with $\mathrm{AD}$ and cerebrovascular disease can mimic the LBD pattern and it's not uncommon to find these pathologies at the same patient (Kemp and Holmes, 2007).

Vascular dementia: Arteriosclerotic brain disease presents as multiple focal areas of hypo perfusion randomly distributed in the cortex, also compromising sub cortical structures. This particular pattern is never been observed in A.D., but the dual pathology is described as being present in $4-10 \%$ of patients. The differential diagnosis is AIDS dementia complex. When cerebrovascular disease is predominantly microangiopathic there is significantly more diffuse cortical hypoperfusion and ventricle enlargement (Pakrasi and O’Brien, 2005).

Front temporal lobar degeneration: Patients with the different clinical subtypes in this group present severe bilateral hypo perfusion in the frontal lobes, predominantly in the mesial structures (Caixeta and Nitrini, 2001). There is also perfusion deficit in the anterior regions of the temporal lobes and each subtype has other characteristic findings, like asymmetrical parietal hypo perfusion in primary progressive aphasia.

Also depressive disorders and transient global amnesia can show prefrontal symmetric hypo perfusion, but there is unilateral basal ganglia hypo perfusion in the later, more commonly in the right. Also Huntington's disease can show bilateral front temporal hypo perfusion, but it presents later in the progression of the disease, while the first sign is severe bilateral basal ganglia hypo perfusion.

Dementia associated with Parkinsonism: As described in Lewy body dementia, idiopathic Parkinson disease with dementia can show hypo perfusion patterns similar to those observed in $\mathrm{AD}$, but basal ganglia hypo perfusion is far more frequent, as is frontal precentral hypo perfusion (Caixeta and Vieira,
2008). Corticobasal degeneration presents as bilateral asymmetrical frontal, parietal and basal ganglia hypo perfusion. Supranuclear palsy also presents frontal hypo perfusion, predominantly in the menial regions, but what specifically distinguishes this disease is symmetric severe basal ganglia hypo perfusion. Multiple system atrophy has a very specific finding, which is cerebella hypo perfusion, besides symmetric basal ganglia hypo perfusion. Hippocampal hypo perfusion with bilateral frontal and parietal hypo perfusion is found in normal pressure hydrocephalus.

\section{Other amnesic syndromes:}

Transient global amnesia: Almost 60 years after its initial description, Transient Global Amnesia (TGA) remains one of the most enigmatic syndromes in clinical neurology. Recent Diffusion-Weighted Imaging (DWI) data suggest that a transient perturbation of hippocampal function is the functional correlate of TGA because focal diffusion lesions can be selectively detected in the CA1 field of the hippocampal cornu ammonias. The vulnerability of CA1 neurons to metabolic stress plays, therefore, a pivotal part in the path physiological cascade, leading to an impairment of hippocampal function during TGA (Bartsch et al., 2006; Bartsch and Deuschl, 2010). The maximum level of detection of these lesions is 24-72 $\mathrm{h}$ after onset of symptoms. Although the diagnosis of TGA is primarily a clinical one, neuroimaging in TGA can positively support the diagnosis.

Although various factors, such as migraine, focal ischemia, venous flow abnormalities and epileptic phenomena, have been suggested to be involved in the path physiology of TGA, the factors triggering the emergence of these lesions are still elusive (Bartsch and Deuschl, 2010). To date, data from MRI has not found evidence for structural squeal of these hippocampal lesions and recent neuropsychological findings have also not found evidence for clinically relevant chronic neuropsychological deficits.

Quantitative imagings of changes of regional cerebral glucose, oxygen metabolism, or cerebrovascular blood flow in TGA have been studied by use of PET and Single Photon Emission Computed Tomography (SPECT). In some studies, mesiotemporal flow changes have been described (Eustache et al., 2000; Warren et al., 2000; Guillery et al., 2002). However, most studies have also noted concomitant decreased or increased changes in cerebral blood flow in other anatomical structures, such as unilateral or bilateral thalamic, prefrontal, frontal, amygdaline, striatal, cerebellar, occipital, precentral and post central areas (Eustache et al., 2000; Nardone et al., 2004). In 
some studies, no mesiotemporal changes, no cerebral changes, or a global hypo perfusion were detected (Chung et al., 2009). In summary, the imaging data derived from PET and SPECT studies are difficult to compare and interpret. Most changes normalized on follow-up examination. The variability's in PET and SPECT are probably associated with differences in the study designs, such as the imaging protocol and resolution and the latency of scanning, frequently done days or weeks after the acute TGA and thus not covering the initial path physiological event. A correlation between SPECT and MRI in the time window of 24-73 $\mathrm{h}$ after onset has been found in a study including only six patients. In five patients, a predominant hypo perfusion in the cerebella vermin was observed in combination with punctuate DWI lesions in the hippocampus, whereas in another patient a bilateral hypo perfusion in the temporal lobes was detected by use of SPECT. Functional MRI was used in two patients during an acute TGA to assess memory function and cerebral activation patterns. In both patients, there was reduced or no activation in temporal lobe structures during encoding of visual scenes or recognition of old scenes, thus reflecting the functional impairment of temporal lobe structures (Bartsch and Deuschl, 2010).

Use of structural imaging with MRI has detected abnormalities in memory-relevant structures of the mesiotemporal region. Early results have been inconsistent and controversial about the type and location of signal abnormalities described in some patients (Matsui et al., 2002; Huber et al., 2002). Recent data from studies that used high-resolution MRI have shown that focal hyper intense lesions correlating to restricted diffusion in the lateral hippocampus can now be reliably detected (Bartsch et al., 2006; 2007). The detection rate of these lesions can be improved by up to $85 \%$ with optimized MRI parameters and by acknowledging the time course of the lesion.

Psychogenic amnesias: Commonly, memory disturbances are related to organic brain damage. Nevertheless, especially the old psychiatric literature provides numerous examples of patients with selective amnesia due to what at that time was preferentially named hysteria (Markowitsch, 2003) and which implied that both environmental circumstances and personality traits influence bodily and brain states to a considerable degree. Awareness of the existence of relations between cognition, soma and psyche has increased especially in recent times and has created the research branch named cognitive neuropsychiatry. Within this branch, psychopathologic processes, deviating from normal information processing, are studied with a combination of methods derived from neurology, psychiatry, psychology, neurobiology and neuroinformatics in order to provide a deeper understanding of psychic disease processes (Halligan and David, 2001).

Modern imaging methods have helped to establish similarities between organic and functional (psychogenic) amnesias with respect to their brain correlates. In fact, it was found that also environmentalrelated amnesias caused by stress and trauma situations with which individuals cannot cope appropriately may alter the brain's metabolism in a predictable way and that even therapy-induced reestablishments of memory may be related to gains in brain metabolism. These results support the view, according to Markowitsch, (1996), that organic and functional amnesias are two sides of the same coin. According this author, both kinds of phenomena may derive from a common brain mechanism leading to blockade, disruption, or disconnection mechanisms affecting the processes of memory and disintegrating widespread memory networks in the brain. This disintegration may be a consequence of "mechanical alterations" in the brain of organic amnesiacs and may be a consequence of biochemical alterations in the brain of psychogenic amnesiacs. For these, such processes may be induced during autobiographical information processing by resynchronizations memory patterns, resulting in the reduction of discomfort for the subject.

Functional amnesias and psychogenic amnesias are discussed and their symptomatology is compared to that of organic amnesias. The term "monastic block syndrome" (Markowitsch, 2003) is introduced and defined as a syndrome of its own. Experimental data, obtained especially with functional imaging methods, are presented to elucidate changes in neural activation during functional amnesic states. Functional amnesic states, confined to a patient's biography, can be triggered by environmentally induced stress and trauma, leading to lasting inability to retrieve autobiographical events. Such impairment may be identified at the brain level using functional imaging techniques (Markowitsch, 2003).

Illusory memories: A key point of agreement between cognitive and biological theories is that memories do not preserve a literal representation of the world; memories are constructed from fragments of information that are distributed across different brain regions and depend on influences operating in the present as well as the past. Memory illusions and distortions have long been of interest to psychology researchers studying memory, but neuropsychologists 
and neuroscientists have paid relatively little attention to them (Schacter, 1996).

Memory distortions and illusions have long been of interest to psychopathology, dating to the classic 1932 study by Bartlett on the reconstructive nature of memory (Baddeley, 2000). Three decades later, Neisser (2004) put forward similar ideas. His monograph stimulated intensive interest on the part of cognitive psychologists in questions concerning memory distortions, resulting in many striking demonstrations of erroneous remembering in laboratory studies. Cognitive studies concerning memory distortion continued through the 1980s and have grown dramatically during the 1990s, inspired in part by controversies over the accuracy of memories retrieved in psychotherapy and effects of suggestive questioning on the reliability of children's' recollections (Schacter, 1996; Peres et al., 2008). Neuroimaging studies have highlighted important issues related to structural and functional brain changes found in sufferers of psychological trauma that may influence their ability to synthesize, categorize and integrate traumatic memories (Peres et al., 2008).

A number of neuropsychologists have noted that damage to the ventromedial aspects of the frontal lobes and basal forebrain are often associated with the memory distortion known as confabulation, where patients describe detailed recollections of events that never happened (Schacter and Coyle, 1997). Schacter and Coyle (1997) has contended that confabulation arises as a result of impairment to strategic retrieval and monitoring processes that depend on frontal regions. Illusory memory conjunctions appear to be attributable to inadequate binding of features at the time of encoding, a process that likely depends on the hippocampal formation.

Schacter et al. (1996) conducted a PET study using a specific paradigm: before scanning, subjects listened to a long list of words that were grouped into semantic categories that each included 20 associates of a nonpresented critical lure; then was administered yes/no recognition tests in separate $60 \mathrm{sec}$ scans for old words that had appeared on the study list ("true targets"), critical lures that had not appeared but were related to previously presented words ("false targets") and new words that had not appeared and were not systematically related to previously presented words ("true target controls" and "false target controls", respectively); in a separate passive fixation scan, subjects simply looked at a crosshair for $60 \mathrm{sec}$. Compared to the passive fixation condition, both accurate recognition of true targets and illusory recognition of false targets were accompanied by significant blood flow increases in many of the same brain regions, including bilateral anterior/dorsolateral frontal cortex, presumes, bilateral cerebellum and the left medial temporal lobe, in the vicinity of the parahippocampal gyros. This latter observation is intriguing in light of previously mentioned findings that amnesic patients with medial temporal damage exhibited little false recognition of critical lures and that medial temporal blood flow increases are associated with successful recollection (Schacter et al., 1995). However, the evidence linking parahippocampal gyrus activation with episodic recognition (as opposed to visual or lexical processing) was equivocal, so this finding must be viewed cautiously pending further research. Direct comparison between veridical and illusory recognition in this study (Schacter et al., 1996) yielded virtually no significant findings, suggesting that brain activity during the two forms of recognition is quite similar. Nonetheless, the authors did observe some suggestive differences. Veridical recognition was accompanied by significantly greater blood flow than illusory recognition in the vicinity of the left supramarginal gyrus and superior temporal gyrus. Previous PET studies have implicated these regions in the processing and storage of phonological information (Paulesu et al., 1993; Muller and Knight, 2006). Moreover, studies of brain-damaged patients have linked the supramarginal gyros with disruptions of phonological analysis and retrieval (Kroll et al., 1996; Reder et al., 2009). In light of these observations, authors hypothesized that temporoparietal increases associated with veridical recognition may reflect subjects' recognition of having heard or rehearsed the target words at the time of study; no such auditory/phonological information was available for critical lures.

One further suggestive finding from this PET study (Schacter et al., 1996) relates to the previously mentioned observations of a link between frontal lobe impairments and heightened susceptibility to false recognition. The authors found that false recognition was associated with trends for greater blood flow increases in inferior frontal regions (orbit frontal cortex) bilaterally and right anterior prefrontal cortex than was veridical recognition. One possible interpretation of this finding is that subjects were trying to oppose or inhibit the sense of familiarity or recollection associated with the critical lures. That is, when subjects were deciding whether a critical lure had appeared previously, they likely experienced a strong feeling that it did. At the same time, knowing that many associatively related items were on the list, they may have engaged in effortful retrieval processes as they tried to remember specific information about the test item's appearance in the study list. 


\section{CONCLUSION}

Dementia is a common illness with an incidence that is rising as the aged population increases. It affects about $8 \%$ of people age 65 years and older. Identification of dementia is particularly difficult in its early phases when family members and physicians often incorrectly attribute the patient's symptoms to normal aging. There are a number of dementias, including Alzheimer's disease, dementia with Lewy bodies and front temporal dementia, which is subdivided into the behavioral variant, the semantic variant and confluent variant. A variety of powerful techniques that have allowed visualization of organ structure and function with exact detail have been introduced in the last twenty-five years. The role of imaging in dementia has traditionally been directed at ruling out treatable and reversible etiologies and not to use imaging to better understand the path physiology of the different dementias. Different brain imaging techniques allow the examination of the structure, biochemistry, metabolic state and functional capacity of the brain. All of the major neurodegenerative disorders have relatively specific imaging findings that can be identified. New imaging techniques carry the hope of revolutionizing the diagnosis of dementia so as to obtain a complete molecular, structural and metabolic characterization, which could be used to improve diagnosis and to stage each patient and follow disease progression and response to treatment. Structural and functional imaging modalities contribute to the diagnosis and understanding of the different dementias. In this review, we presented current opportunities of neuroimaging techniques in the diagnosis and differentiation of neurodegenerative disorders.

\section{REFERENCES}

Aine, C.J., L. Sanfratello, J.C. Adair, J.E. Knoefel and A. Caprihan et al., 2011. Development and decline of memory functions in normal, pathological and healthy successful aging. Brain Topogr., 24: 323-339. DOI: $10.1007 / \mathrm{s} 10548-011-0178-\mathrm{x}$

Apostolova, L.G. and P.M. Thompson, 2008. Mapping progressive brain structural changes in early Alzheimer's disease and mild cognitive impairment. Neuropsychologia, 46: 1597-1612. DOI: 10.1016/j.neuropsychologia.2007.10.026

Baddeley, A., 2000. The episodic buffer: A new component of working memory? Trends Cogn. Sci., 4: 417-423. DOI: 10.1016/S13646613(00)01538-2
Bartsch, T. and G. Deuschl, 2010. Transient global amnesia: Functional anatomy and clinical implications. Lancet Neurol., 9: 205-214. DOI: 10.1016/S1474-4422(09)70344-8

Bartsch, T., K. Alfke, G. Deuschl and O. Jansen, 2007. Evolution of hippocampal CA-1 diffusion lesions in transient global amnesia. Ann. Neurol., 62: 475-480. DOI: 10.1002/ana.21189

Bartsch, T., K. Alfke, R. Stingele, A. Rohr and S. Freitag-Wolf et al., 2006. Selective affection of hippocampal CA-1 neurons in patients with transient global amnesia without long-term sequelae. Brain., 129: 2874-2884. DOI: 10.1093/brain/awl248

Burns, M.S. and J. Fahy, 2010. Broca's area: rethinking classical concepts from a neuroscience perspective. Top. Stroke Rehabil., 17: 401-410. PMID: 21239364

Caixeta, L. and R. Nitrini, 2001. Subtipos clínicos da demência frontotemporal. Arq. Neuro-Psiquiatr., 59: $\quad 577-581$. DOI: $10.1590 /$ S0004282X2001000400017

Caixeta, L. and R.T. Vieira 2008. Demência na doença de Parkinson. Rev. Bras Psiquiatr., 30: 375-383. DOI: $10.1590 / \mathrm{S} 1516-44462008005000016$

Cappa, S.F., 2011. The neural basis of aphasia rehabilitation: Evidence from neuroimaging and neurostimulation. Neuropsychol. Rehabil., 21: 742754. DOI: 10.1080/09602011.2011.614724

Caserta, M.T., Y. Bannon, F. Fernandez, B. Giunta and M.R. Schoenberg et al., 2009. Normal brain aging clinical, immunological, neuropsychological and neuroimaging features. Int. Rev. Neurobiol., 84: 119. DOI: $10.1016 / \mathrm{S} 0074-7742(09) 00401-2$

Celsis, P., 2000. Age-related cognitive decline, mild cognitive impairment or preclinical Alzheimer's disease? Ann. Med., 32: 6-14. PMID: 10711572

Cheng, K. and C.H. Zou, 2007. Biomedicine and informatics model of alzheimer's disease. Am. J. Biochem. Biotechnol., 3: 145-149. DOI: 10.3844/ajbbsp.2007.145.149

Chung, Y.A., J. Jeong, D.W. Yang, B.J. Kang and S.H. Kim et al., 2009. A Tc-99m SPECT study of regional cerebral blood flow in patients with transient global amnesia. Neuroimage, 47: 50-55. DOI: 10.1016/j.neuroimage.2008.11.011

Degenszajn, J., P. Caramelli, L. Caixeta and R. Nitrini, 2001. Encoding process in delayed recall impairment and rate of forgetting in Alzheimer's disease. Arq. Neuropsiquiatr., 59: 171-174. PMID: 11400019

D'Esposito, M., 2000. Functional Imaging of Neurocognition. Semin. Neurol., 20: 487-498. 
Devous, M.D., 2002. Functional brain imaging in the dementias: Role in early detection, differential diagnosis and longitudinal studies. Eur. J. Nuc. Med. Mol. Imag., 29: 1685-1696. DOI: 10.1007/s00259-002-0967-2

Dickerson, B.C., D.H. Salat, J.F. Bates, M. Atiya and R.J. Killiany et al., 2004. Medial temporal lobe function and structure in mild cognitive impairment. Ann. Neurol., 56: 27-35. DOI: 10.1002/ana.20163

Dronkers, N.F., 1996. A new brain region for coordinating speech articulation. Nature, 384: 159161. PMID: 8906789

Eustache, F., B. Desgranges, M. Aupee, B. Guillery and J.C. Baron, 2000. Functional neuroanatomy of amnesia: positron emission tomography studies. Microsc. Res. Tech., 51: 94-100. DOI: 10.1002/1097-0029(20001001)51:1<94::AIDJEMT10>3.0.CO;2-P

Fayed, N., S. Olmos, H. Morales and P.J. Modrego, 2006. Physical basis of magnetic resonance spectroscopy and its application to central nervous system diseases. Am. J. Applied Sci., 3: 18361845. DOI: 10.3844/ajassp.2006.1836.1845

Guillery, B., B. Desgranges, V. de la Sayette, B. Landeau and F. Eustache et al., 2002. Transient global amnesia: concomitant episodic memory and positron emission tomography assessment in two additional patients. Neurosci. Lett., 325: 62-66. DOI: 10.1016/S0304-3940(02)00233-1

Halligan, P.W. and A.S. David, 2001. Cognitive neuropsychiatry: towards a scientific psychopathology. Nat. Rev. Neurosci., 2: 209-215. DOI: 10.1038/35058586

Hamalainen, A., M. Pihlajamaki, H. Tanila, T. Hanninen and E. Niskanen et al., 2007. Increased fMRI responses during encoding in mild cognitive impairment. Neurobiol. Aging., 28: 1889-1903. DOI: 10.1016/j.neurobiolaging.2006.08.008

Heertum, R.L.V. and R.S. Tikofsky, 2003. Positron emission tomography and single-photon emission computed tomography brain imaging in the evaluation of dementia. Semin. Nucl. Med., 33: 7785. PMID: 12605358

Herholz, K., 2003. PET studies in dementia. Ann. Nucl. Med., 17: 79-89. DOI: 10.1007/BF02988444

Herholz, K., P. Herscovitch and W.D. Heiss, 2004. NeuroPET: Positron Emission Tomography in Neuroscience and Clinical Neurology. 1st Edn., Springer-Verlag, Berlin, New York, ISBN 9783540006916, pp: 297.
Huber, R., A.J. Aschoff, A.C. Ludolph and M.W. Riepe, 2002. Transient global amnesia. Evidence against vascular ischemic etiology from diffusion weighted imaging. J. Neurol., 249: 1520-1524. DOI: $10.1007 / \mathrm{s} 00415-002-0881-3$

Jak, A.J, K.J. Bangen, C.E. Wierenga, L. Delano-Wood and J. Corey-Bloom et al., 2009. Contributions of neuropsychology and neuroimaging to understanding clinical subtypes of mild cognitive impairment. Int. Rev. Neurobiol., 84: 81-103. DOI: 10.1016/S0074-7742(09)00405-X

Johnson, S.C., L.C. Baxter, L. Susskind-Wilder, D.J. Connor and M.N. Sabbagh et al., 2004. Hippocampal adaptation to face repetition in healthy elderly and mild cognitive impairment. Neuropsychologia, 42: 980-989. DOI: 10.1016/j.neuropsychologia.2003.11.015

Johnson, S.C., T.W. Schmitz, C.H. Moritz, M.E. Meyerand and H.A. Rowley et al., 2006. Activation of brain regions vulnerable to Alzheimer's disease: The effect of mild cognitive impairment. Neurobiol. Aging., 27: 1604-1612. DOI: 10.1016/j.neurobiolaging.2005.09.017

Kalpouzos, G., F. Eustache and B. Desgranges, 2008. Cognitive reserve and neural networks in normal aging and Alzheimer's disease. Psychol. Neuropsychiatr. Viei., 6: 97-105. PMID: 18556268

Kemp, P.M. and C. Holmes, 2007. Imaging in dementia with Lewy bodies: A review. Nucl. Med. Commun., 28: 511-519. PMID: 17538391

Kircher, T.T., S. Weis, K. Freymann, F. Jessen and W. Grodd et al., 2007. Hippocampal activation in patients with mild cognitive impairment is necessary for successful memory encoding. J. Neurol. Neurosurg Psychiatry, 78: 812-818. DOI: 10.1136/jnnp.2006.104877

Kroll, N.E.A., R.T. Knight, J. Metcalfe, E.S. Wolf and E. Tulving, 1996. Cohesion failure as a source of memory illusions. J. Mem. Language, 35: 176-196.

Lee, B.C., M. Mintun, R.L. Buckner and J.C. Morris, 2003. Imaging of Alzheimer's disease. J. Neuroimag., 13: 199-214. DOI: 10.1111/j.15526569.2003.tb00179.x

Lopes, H.F.S., J.M. Abe, P.A.M. Kanda, S. Machado and B. Velasques et al., 2011. Improved application of paraconsistent artificial neural networks in diagnosis of Alzheimer's disease. Am. J. Neurosci., 2: 54-64. DOI: 10.3844/amjnsp.2011.54.64

Mandzia, J.L., M.P. McAndrews, C.L. Grady, S.J. Graham and S.E. Black, 2009. Neural correlates of incidental memory in mild cognitive impairment: An fMRI study. Neurobiol. Aging, 30: 717-730. DOI: 10.1016/j.neurobiolaging.2007.08.024 
Markowitsch, H.J., 1996. Organic and psychogenic retrograde amnesia: Two sides of the same coin? Neurocase, 2: 357-371. DOI: $10.1080 / 13554799608402410$

Markowitsch, H.J., 2003. Psychogenic amnesia. Neuroimage, 20: S132-S138. DOI: 10.1016/j.neuroimage.2003.09.010

Matsui, M., T. Imamura, S. Sakamoto, K. Ishii and H. Kazui et al., 2002. Transient global amnesia: increased signal intensity in the right hippocampus on diffusion-weighted magnetic resonance imaging. Neuroradiology, 44: 235-238. DOI: $10.1007 / \mathrm{s} 002340100699$

Mazziotta, J.C. and M.E. Phelps, 1984. Human sensory stimulation and deprivation: Positron emission tomographic results and strategies. Ann. Neurol., 15: 50-60. DOI: 10.1002/ana.410150711

Miller, S.L., E. Fenstermacher, J. Bates, D. Blacker and R.A. Sperling et al., 2008. Hippocampal activation in adults with mild cognitive impairment predicts subsequent cognitive decline. J. Neurol. Neurosurg. Psychiatry, 79: 630-635. PMID: 17846109

Mosconi, L., 2005. Brain glucose metabolism in the early and specific diagnosis of Alzheimer's disease. FDG-PET studies in MCI and AD. Eur. J. Nucl. Med. Mol. Imag., 32: 486-510. PMID: 15747152

Mosconi, L., M. Brys, L. Glodzik-Sobanska, S. De Santi and H. Rusinek et al., 2007. Early detection of Alzheimer's disease using neuroimaging. Exp. Gerontol., 42: 129-138. DOI: 10.1016/j.exger.2006.05.016

Muller, N.G. and R.T Knight, 2006. The functional neuroanatomy of working memory: Contributions of human brain lesion studies. Neuroscience, 139: 51-58. DOI: 10.1016/j.neuroscience.2005.09.018

Nardone, R., E.C. Buffone, M.F. Matullo and F. Tezzon, 2004. Motor cortex excitability in transient global amnesia. J. Neurol., 251: 42-46. DOI: $10.1007 / \mathrm{s} 00415-004-0270-1$

Neisser, U., 2004. Memory development: New questions and old. Dev. Rev., 24: 154-158. DOI: 10.1016/j.dr.2003.09.002

Nitrini, R., C.A. Buchpiguel, P. Caramelli, V.S. Bahia and S.C. Mathias et al., 2000. SPECT in Alzheimer's disease:features associated with bilateral parietotemporal hypoperfusion. Acta. Neurol. $\quad$ Scand., 101: 172-176. DOI: 10.1034/j.1600-0404.2000.101003172.x

Nordberg, A., 2004. PET imaging of amyloid in Alzheimer's disease. Lancet. Neurol., 3: 519-527. DOI: $10.1016 / \mathrm{S} 1474-4422(04) 00853-1$
Pakrasi, S. and J.T. O'Brien, 2005. Emission tomography in dementia. Nucl. Med. Commun., 26: 189-196. PMID: 15722899

Paulesu, E., C.D. Frith and R.S.J. Frackowiak, 1993. The neural correlates of the verbal component of working memory. Nature, 362: 342-345. DOI: 10.1038/362342a0

Peres, J.F., A. McFarlane, A.G. Nasello and K.A. Moores, 2008. Traumatic memories: Bridging the gap between functional neuroimaging and psychotherapy. Aust. N. Z. J. Psychiatry, 42: 478488. PMID: 18465374

Petersen, S.E., P.T. Fox, M.I. Posner, M. Mintun and M.E. Raichle, 1988. Positron emission tomographic studies of the cortical anatomy of single-word processing. Nature, 331: 585-589. PMID: 3277066

Pievani, M., W.d. Haan, T. Wu, W.W. Seeley and G.B. Frisoni, 2011. Functional network disruption in the degenerative dementias. Lancet Neurol., 10: 829-843. DOI: 10.1016/S1474-4422(11)70158-2

Rajah, M.N. and M. D'Esposito, 2005. Region-specific changes in prefrontal function with age: A review of PET and fMRI studies on working and episodic memory. Brain, 128: 1964-1983. DOI: 10.1093/brain/awh608

Reder, L.M., H. Park and P.D. Kieffaber, 2009. Memory systems do not divide on consciousness: Reinterpreting memory in terms of activation and binding. Psychol. Bull., 135: 23-49. PMID: 19210052

Rossini, P.M. and G.D. Forno, 2004. Integrated technology for evaluation of brain function and neural plasticity. Phys. Med. Rehabil. Clin. N. Am., 15: 263-306.

Schacter, D.L and J.T. Coyle, 1997. Memory Distortion: How Minds, Brains and Societies Reconstruct the Past. 1st Edn., Harvard University Press, Cambridge, MA., ISBN 9780674566767, pp: 417.

Schacter, D.L., 1996. Illusory memories: A cognitive neuroscience analysis. Proc. Natl. Acad. Sci., 93: 13527-13533.

Schacter, D.L., E. Reiman, A. Uecker, M.R. Polster and L.S. Yun et al., 1995. Brain regions associated with retrieval of structurally coherent visual information. Nature, 376: 587-590. PMID: 7637806

Schacter, D.L., E. Reiman, T. Curran, L.S. Yun and D. Bandy et al., 1996. Neuroanatomical correlates of veridical and illusory recognition memory: Evidence from positron emission tomography. Neuron, 17: 267-274. DOI: 10.1016/S08966273(00)80158-0 
Silverman, D.H.S., 2004. Brain ${ }^{18}$ F-FDG PET in the diagnosis of neurodegenerative dementias: Comparison with perfusion SPECT and with clinical evaluations lacking nuclear imaging. J. Nuc. Med., 45: 594-607.

Sperling, R., 2007. Functional MRI studies of associative encoding in normal aging, mild cognitive impairment, and Alzheimer's disease. Ann. N. Y. Acad. Sci., 97: 146-155. PMID: 17413017

Vannini, P., O. Almkvist, T. Dierks, C. Lehmann and L.O. Wahlund, 2007. Reduced neuronal efficacy in progressive mild cognitive impairment: A prospective fMRI study on visuospatial processing. Psychiatry Res., 156: 43-57. DOI: 10.1016/j.pscychresns.2007.02.003
Walters-Williams, J. and Y. Li, 2011. Using invariant translation to denoise electroencephalogram signals. Am. J. Applied Sci., 8: 1122-1130. DOI: 10.3844/ajassp.2011.1122.1130

Warren, J.D., B. Chatterton, P.D. Thompson, 2000. A SPECT study of the anatomy of transient global amnesia. J. Clin. Neurosci., 7: 57-59. DOI: 10.1054/jocn.1998.0129 